\title{
Recommendations for the reporting of lymphoid neoplasms: A report from the Association of Directors of Anatomic and Surgical Pathology
}

\section{Elaine S Jaffe ${ }^{1}$, Peter M Banks ${ }^{2}$, Bharat Nathwani ${ }^{3}$, Jonathan Said ${ }^{4}$ and Steven H Swerdlow ${ }^{5}$} The Ad Hoc Committee on reporting of lymphoid neoplasms

${ }^{1}$ Laboratory of Pathology, National Cancer Institute, Bethesda, MD, USA; ${ }^{2}$ Carolinas Medical Center, Charlotte, NC, USA; ${ }^{3}$ University of Southern California School of Medicine, Los Angeles, CA, USA; ${ }^{4} U C L A$ Medical Center for the Health Sciences, Los Angeles, CA, USA and ${ }^{5}$ University of Pittsburgh Medical Center, Pittsburgh, PA, USA

\begin{abstract}
In this report, the Association of Directors of Anatomic and Surgical Pathology (ADASP) provides guidelines for the reporting of lymphoid neoplasms. The World Health Organization Classification of Tumors of the Haematopoietic and Lymphoid Tissues is the preferred international standard for diagnostic criteria (disease definition) and nomenclature. Ancillary studies are often required, and the Association recommends that immunophenotypic and genotypic information be integrated into the final report, to the extent possible.

Modern Pathology (2004) 17, 131-135, advance online publication, 5 December 2003; doi:10.1038/modpathol.3800028
\end{abstract}

Keywords: Lymph node; lymphoma; Immunohistochemistry; molecular diagnostics; quality assurance; laboratory management

The Association of Directors of Anatomic and Surgical Pathology (ADASP) has named several committees to develop recommendations regarding the content of the surgical pathology report for common malignant tumors. A committee of individuals with special interest and expertise write the recommendations, which are reviewed by the council of ADASP and subsequently by the entire membership.

The recommendations have been divided into the following four major areas: (1) items that provide an informative gross description; (2) additional diagnostic features that are recommended to be included in every report if possible; (3) optional features that may be included in the final report; and (4) a checklist.

\section{Features the Association recommends to be included in the final report}

Background Clinical Information

The Association recommends the inclusion of pertinent clinical history, when this information is

Correspondence: Dr ES Jaffe, Hematopathology Section, Laboratory of Pathology, NCI, Building 10/Room 2N202, 10 Center Drive MSC-1500, National Institutes of Health, Bethesda, MD 208921500, USA.

E-mail: elainejaffe@nih.gov

Received 25 August 2003; accepted 16 October 2003; published online 5 December 2003 available. The pathologist is encouraged to obtain clinical history, if possible. For some diseases, an accurate history may be essential to diagnosis, for example, post-transplant-associated lymphoproliferative disease.

1. Previous diagnosis of a lymphoid neoplasm, if known. Specify dates and site(s), and treatment status, if available.

2. Presence of generalized or localized lymphadenopathy.

3. Evidence of organomegaly (eg, hepatosplenomegaly).

4. Pertinent hematological findings (eg, lymphocytosis, pancytopenia).

5. Constitutional symptoms.

6. HIV status.

7. Prior immune abnormality, including congenital immune disorders.

8. Autoimmune disease.

9. Other pertinent serology (eg, HTLV-I, EpsteinBarr virus).

10. Other known cofactors, (eg, Helicobacter pylori infection).

\section{Gross Description}

The proper handling of the lymph node biopsy specimen is critical to ensure proper fixation, which is essential for the preparation of high-quality 
histological sections. ${ }^{1}$ The Association recommends that the pathologist receive lymph node biopsies fresh, intact, and that an unsectioned lymph node biopsy should never be immersed in fixative. It is recommended that each laboratory establish a protocol for the handling of lymph node biopsies that ensures both optimal histological sections and preservation of material for ancillary studies. These principles, and the procedures outlined below, also apply to extranodal sites that may be biopsied or resected for a potential diagnosis of lymphoma.

1. Identification: State how the specimen was identified: labeled with the patient's name, medical record number, organ or site.

2. State how the specimen was received (fresh, in fixative, intact vs sectioned).

3. State the surgical procedure used to procure the specimen (excisional biopsy vs incisional, vs core biopsy).

4. State the dimensions of the specimen.

5. State if there is a capsule, and if it is intact or altered grossly.

6. Describe the color and consistency (firm vs fleshy); presence of nodularity, necrosis, hemorrhage.

7. The Association recommends that lymph nodes be sectioned at $2 \mathrm{~mm}$ intervals, to ensure appropriate fixation. ${ }^{1}$

8. If the size of the lymph node permits it, it is preferable to cut sections perpendicular to the long axis of the lymph node. This orientation provides the greatest assessment of the architecture.

9. If the specimen is a spleen, provide the weight. Describe the appearance of any focal lesions (eg, infarcts, nodules, hemorrhage), and gross abnormalities of red or white pulp.

10. If the specimen is a spleen obtained for staging, the Association recommends that the spleen be sectioned at $3-5 \mathrm{~mm}$ intervals, to look for grossly identifiable lesions. First fixing thicker slices $(1 \mathrm{~cm})$ briefly in formalin may facilitate sectioning at the desired thickness. For staging laparotomy specimens for Hodgkin's lymphoma, the number of grossly identifiable lesions, if less than 10, should be stated. The presence of more than four nodules has been shown to be of prognostic significance. ${ }^{2}$

11. Unique identifiers should be used for each cassette, and the gross description should also specify the type of fixation used for each paraffin block.

12. It is often desirable to fix tissue in more than one fixative. Some fixatives provide excellent cytological detail (B5, B + ), but compromise the ability to extract DNA for molecular studies. Formalin is most suitable if polymerase chain reaction (PCR) studies from the paraffin-embedded sections are anticipated.

13. Snap-freezing is useful for preserving tissue for frozen-section immunohistochemistry, or future molecular studies. The following is a suitable procedure.

(a) One or more blocks of fresh tissue approximately $1.0 \times 1.0 \times 0.3 \mathrm{~cm}^{3}$ are cut from the specimen.

(b) The tissue blocks are placed in a mold, cork or other suitable form and immersed in Optimal Cutting Temperature (OCT) (Sakura Tissue Tek; Torrance, CA, USA) embedding compound.

(c) A sludge is made of dry ice and isopentane (2methyl butane) and the tissue and mold are immersed into the solution and snap-frozen.

(d) The blocks are labeled and stored at $-80^{\circ} \mathrm{C}$ or over liquid nitrogen.

(e) Blocks in OCT are suitable for frozen-section immunohistochemistry and also can be used for molecular analyses. If there is sufficient tissue, blocks can be snap-frozen without OCT for molecular studies and held at $-80^{\circ} \mathrm{C}$ or over liquid nitrogen until needed.

\section{Diagnostic Information}

1. Specify exact anatomic site, if known, and tissue (lymph node or other).

2. Specify procedure (excisional biopsy, incisional biopsy, needle core).

3. Histological tumor type. The Association recommends the use of the WHO classification of tumors of the hematopoietic and lymphoid tissues (Tables $1-4){ }^{3}$ The designation of morphologic or clinical variants is considered optional for most clinical purposes. If an alternative classification scheme is used, it should be so specified in the diagnosis.

4. Specify if the specimen is only focally or incompletely involved.

5. Specify if more than one histologic type is identified ie, composite lymphoma, or progression to lymphoma of higher histologic grade).

6. Specimen adequacy. A precise diagnosis may not be possible in some instances due to limitations of specimen adequacy (eg, needle biopsy, necrotic or fibrotic specimen). If specimen adequacy is of concern, this fact should be stated explicitly.

7. If ancillary studies (eg, immunocytochemistry, molecular diagnostics) were performed, the diagnosis or comment should contain a statement regarding these studies and their diagnostic implications (Table 5).

\section{Features that may be optional in the final report}

\section{Immunophenotypic Information}

For many subtypes of lymphoid neoplasms (eg, peripheral T-cell lymphomas, diffuse large B-cell lymphomas, precursor B-cell lymphoblastic lymphoma/leukemia), immunophenotypic studies are 
Table 1 WHO classification of B-cell lymphoid neoplasms

Precursor B-cell neoplasm

Precursor B-lymphoblastic leukemia/lymphoma

Mature B-cell neoplasms

Chronic lymphocytic leukemia/small lymphocytic lymphoma Variant: with plasmacytoid differentiation or monoclonal gammopathy

B-cell prolymphocytic leukemia

Lymphoplasmacytic lymphoma

Splenic marginal zone B-cell lymphoma ( \pm villous lymphocytes)

Hairy cell leukemia

Variant: hairy cell variant

Plasma cell myeloma/plasmacytoma

Extranodal marginal zone B-cell lymphoma of MALT type

Nodal marginal zone B-cell lymphoma ( \pm monocytoid B cells)

Follicular lymphoma

Grading

Grade 1: 0-5 centroblasts/hpf

Grade 2: 6-15 centroblasts/hpf

Grade 3: >15 centroblasts/hpf

Grade 3a: $>15$ centroblasts, but centrocytes are still present Grade 3b. > centroblasts form solid sheets with no residual centrocytes

Variants

Cutaneous follicle center lymphoma

Diffuse follicle center lymphoma

Grade 1: 0-5 centroblasts/hpf

Grade 2: 6-15 centroblasts/hpf

Mantle cell lymphoma

Variant: blastoid

Diffuse large B-cell lymphoma

Subtypes

Mediastinal large B-cell lymphoma

Intravascular large B-cell lymphoma

Primary effusion lymphoma

Morphologic variants

Centroblastic

Immunoblastic

Anaplastic large B-cell

T-cell/histiocyte-rich

Plasmablastic

Lymphomatoid granulomatosis-type

Burkitt's lymphoma/Burkitt's cell leukemia

Morphologic variants

Classical

Atypical

With plasmacytoid differentiation (AIDS-associated)

Subtypes (clinical and genetic)

Endemic

Sporadic

Immunodeficiency-associated

B-cell proliferations of uncertain malignant potential Lymphomatoid granulomatosis (grades 1, 2 and 3)

Post-transplant lymphoproliferative disease

essential to accurate diagnosis., ${ }^{4,5}$ In some instances, immunophenotypic studies may not be required (eg, many instances of follicular lymphoma). If immunophenotypic studies are performed, we recommend that the results be included in an integrated single report (Table 6). ${ }^{6}$ If ancillary studies are performed in a reference laboratory, the results should be discussed in an integrated report, and the reference laboratory report appended.

1. State how immunophenotypic studies were performed (flow cytometry vs immunohistochemistry).
Table 2 WHO classification of T-cell and NK-cell lymphoid neoplasms

Precursor T-cell neoplasm

Precursor T-lymphoblastic lymphoma/leukemia

Mature (peripheral) T-cell and NK-cell neoplasms

T-cell prolymphocytic leukemia

Morphologic variants: small cell, cerebriform cell

T-cell granular lymphocytic leukemia

Aggressive NK-cell leukemia

Blastic 'NK-cell' lymphoma ${ }^{a}$

Adult T-cell leukemia/lymphoma (HTLV-1+)

Clinical variants

Acute

Lymphomatous

Chronic

Smoldering

Hodgkin-like

Extranodal NK/T-cell lymphoma, nasal type

Enteropathy-type T-cell lymphoma

Hepatosplenic T-cell lymphoma

Subcutaneous panniculitis-like T-cell lymphoma

Mycosis fungoides/Sezary syndrome

Variants

Pagetoid reticulosis

MF-associated follicular mucinosis

Granulomatous slack skin disease

Primary cutaneous CD30+T-cell lymphoproliferative disorder Variants

Lymphomatoid papulosis (type A and B)

Primary cutaneous anaplastic large-cell lymphoma

Borderline lesions

Peripheral T-cell lymphoma, not otherwise characterized

Morphologic variants: lymphoepithelioid (Lennert's), T-zone Angioimmunoblastic T-cell lymphoma

Anaplastic large cell lymphoma, (ALK+/ ALK-)

Morphologic variants: lymphohistiocytic, small cell

${ }^{\mathrm{a}}$ Neoplasm of uncertain lineage and stage of differentiation.

For clinical purposes not considered a neoplasm, of uncertain malignant potential.

2. State if immunohistochemistry was performed on paraffin sections or frozen sections.

3. Specify all markers that were investigated, both positive and negative.

4. It is recommended that antigens usually be identified by the CD nomenclature. Use of the common or commercial name is optional, but may be important in some cases as different antibodies to the same CD antigen may show varying sensitivities and specificities (eg, CD20: L26 vs Leu 16).

5. Avoid the use of generic identifiers (eg, B-cell marker, T-cell marker).

6. Specify which population is expressing the antigen.

7. Specify if the antigen is only focally expressed. It may be helpful in some instances to provide an approximation of the percentage of positive cells.

8. Specify where the studies were performed, if not carried out in the local laboratory. 
Table 3 WHO classification of Hodgkin's lymphoma (Hodgkin's disease)

Nodular lymphocyte predominant Hodgkin's lymphoma

Classical Hodgkin's lymphoma

Nodular sclerosis Hodgkin's lymphoma (grades 1 and 2)

Lymphocyte-rich classical Hodgkin's lymphoma

Mixed cellularity Hodgkin's lymphoma

Lymphocyte-depleted Hodgkin's lymphoma

Table 4 Categories of post-transplant lymphoproliferative disorders (PTLD)

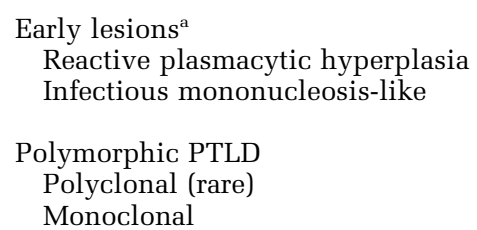

Monomorphic PTLD (classify according to WHO classification)

\section{B-cell lymphomas}

Diffuse large B-cell lymphoma

Burkitt's lymphoma/atypical Burkitt's lymphoma variant

Plasma cell myeloma

\section{T-cell lymphomas}

Peripheral T-cell lymphoma, not otherwise categorized

Other types (hepatosplenic, gamma-delta, NK/T-cell)

Other types (rare)

Hodgkin's lymphoma-like lesions (associated with methotrexate and other immunosuppressive therapy) Hodgkin's lymphoma

Plasmacytoma-like lesions

${ }^{\mathrm{a}}$ Not considered a neoplasm.

9. A discussion of the significance of the immunophenotypic studies is recommended.

\section{Molecular Genetic Studies}

Molecular genetic studies may provide useful diagnostic information about the clonality of the lymphoid infiltrate, the lineage of the lymphoid cells, or a precise molecular abnormality associated with a specific disease. As with immunophenotypic studies, if molecular analysis is performed, the Association recommends that this information be discussed in the context of the histological findings, if possible. Important information to include is:

1. Type of specimen used for the study (frozen tissue vs paraffin-embedded specimen).

2. Specify the method used: PCR vs Southern blot vs RT-PCR, vs others.

3. Specify the exact type of test performed, for example, VJ-PCR for IgH gene rearrangement.

4. Specify if the studies were performed in a reference laboratory or in the local laboratory.

5. Specify the result (monoclonal, polyclonal, oligoclonal) and its possible diagnostic significance.
Table 5 Checklist for the reporting of lymphoid neoplasms

Demographics:

Patient's name:

Age:

Sex:

Race (optional):

Case number:

Clinical history

Prior diagnosis:

Presenting sites of disease:

Clinical symptoms:

Findings on physical examination:

Laboratory findings:

Gross Assessment

Labeling of specimen:

Condition of specimen on receipt:

Fresh:

In fixative:

Intact:

Previously sectioned:

Surgical procedure:

Dimensions of the specimen:

Weight of specimen (if relevant):

Capsule:

Color and consistency:

Focal lesions:

Photography:

Allocation of tissue for special studies:

Frozen tissue for archival storage or other studies

Fresh tissue/cells for flow cytometry, cytogenetics

Other tissue distribution:

Diagnostic information

Anatomic site:

Tissue (lymph node or other):

Histological tumor type:

WHO classification (see Tables 1-4):

Other classification scheme:

Grading (if relevant, ie, follicular lymphoma):

Adequacy:

Focal involvement:

Multiple histological types present (composite lymphoma):

Immunophenotypic data:

Genotypic data:

Cytogenetics:

Microbiological studies:

Special studies

Flow cytometry:

Immunohistochemistry:

Frozen sections:

Paraffin sections:

Molecular genetic studies:

In situ hybridization

PCR:

RT-PCR:

Southern blot:

Cytogenetic studies:

Conventional cytogenetics:

Fluorescence in situ hybridization:

\section{Viral Studies}

Viruses are important cofactors for many lymphoma types, and corroboration of a viral association may be essential for the diagnosis of some diseases, such as adult T-cell leukemia/lymphoma (HTLV-I) or primary effusion lymphoma (HHV-8/KSHV). The pathologist should state the method of identifica- 
Table 6 Example of specimen report

Specimen: Right cervical lymph node (excisional biopsy).

Clinical diagnosis and history: The patient is a 63-year-old female with a history of follicular lymphoma in 1993, treated with chemotherapy, and now presenting with an enlarging right cervical node.

Final diagnosis: Lymph node, right cervical (excisional biopsy): diffuse large B-cell lymphoma (75\%) and follicular lymphoma, grade 2 of $3(25 \%)$ (see comment).

Comment: Slides from the previous lymph node biopsy were reviewed (S93-XXXX) and show follicular lymphoma, grade 1 of 3 . The current specimen shows a grade 2 follicular lymphoma in about $25 \%$ of the node, but in the remainder there is progression to diffuse large B-cell lymphoma. Molecular and cytogenetic studies were not performed in this case. Immunophenotypic studies are consistent with the histological findings, and show positivity for bcl-2 protein in both follicular and diffuse areas. p53 is positive in the diffuse large B-cell lymphoma component. p53 mutations and overexpression have been associated with histological progression in approximately one-third of follicular lymphomas. (Ref: Sander CA, Yano T, Clark HM, et al p53 mutation is associated with progression in follicular lymphomas. Blood 1993;82(7):1994-2004.)

Microscopic description: Nodal architecture is completely effaced with extension beyond the node into the perinodal fat. About $25 \%$ of the node is nodular, with follicles comprising small cleaved cells (centrocytes) as well as large lymphoid cells (centroblasts) with an average of 6-10 large cells per high-power field. The remainder of the node is effaced by a diffuse proliferation of large lymphoid cells with two to three basophilic nucleoli, round or oval nuclear outlines, and pale cytoplasm. Mitoses are rare in the follicles, but frequent in the diffuse large-cell lymphoma. Necrosis is present in about $10 \%$ of the node.

Immunohistochemistry report: Immunoperoxidase stains were performed on B5 fixed sections with appropriate controls.

The atypical lymphoid cells in both the follicular and diffuse areas were positive for CD45, CD20, CD10, bcl-2 and bcl-6 consistent with a B-cell lymphoma of follicle center origin. Ki67 (proliferation marker) is positive in $30 \%$ of the cells in the diffuse areas, and $10 \%$ in the follicular areas. CD3 and CD5 stain admixed small lymphocytes, mainly in the interfollicular regions. p53 is focally positive in follicular areas $(<10 \%$ of follicles stain), but strongly positive in the diffuse large cell component ( $>90 \%$ positive cells).

Gross description: Received fresh from the operating room in normal saline and labeled with the patient's name and hospital number is an excisional biopsy of an intact oval lymph node and adjacent adipose tissue that measures $2.3 \mathrm{~cm} \times 1.4 \mathrm{~cm} \times 1.4 \mathrm{~cm}$. The cut surface is tan and fleshy with foci of necrosis. There is no nodularity grossly visible. The lymph node is serially sectioned at $0.3 \mathrm{~cm}$ intervals and appears homogeneous apart from foci of necrosis. A well-defined capsule is not grossly identified. Imprints are made from the cut surface of the node and air-dried. One slice is snap-frozen in isopentane and dry ice, embedded in OCT, for potential molecular or immunohistochemical studies. A portion of sterile node is placed in culture medium for potential cytogenetic studies or flow cytometry. The remainder of the node is submitted in its entirety in three paraffin blocks, after fixation as follows: A, B5; B formalin; C, formalin.

tion, results (positive or negative), and which cell population is affected for Methods \#1 and \#2.

1. Immunohistochemical stain.

2. In situ hybridization.

3. PCR.

4. Serology (see clinical history).

\section{Cytogenetic Studies}

Cytogenetic studies may provide ancillary diagnostic information useful in the diagnosis or subclassification of lymphoma. The identification of a clonal cytogenetic abnormality supports a diagnosis of malignancy. Some cytogenetic abnormalities are highly associated with specific lymphoid malignancies, for example $t(14 ; 18)$ with follicular lymphoma. The Association recommends that the cytogenetic data be discussed in the context of the histological findings and the complete report appended to the Surgical Pathology report.

1. Conventional cytogenetics.

2. Fluorescence in situ hybridization.

\section{References}

1 Banks PM. Technical aspects of specimen preparation and introduction to special studies (Chapter 1). In: Jaffe ES (ed). Surgical Pathology of the Lymph Nodes and Related Organs, 2nd ed. W.B. Saunders: Philadelphia, PA, 1995, pp. 1-21.

2 Hoppe RT, Rosenberg SA, Kaplan HS, et al. Prognostic factors in pathological stage IIIA Hodgkin's disease. Cancer 1980;46:1240-1246.

3 Jaffe ES, Harris NL, Stein H, et al. Pathology and Genetics of Tumours of Haematopoietic and Lymphoid Tissues. Lyon, France, IARC Press, 2001.

4 The non-Hodgkin's lymphoma classification project: a clinical evaluation of the International Lymphoma Study Group classification of non-Hodgkin's lymphoma. Blood 1997;89:3909-3918.

5 Jaffe ES, Raffeld M. Malignancies of the lymphoid and histiocytic systems. In: Rose NR, Hamilton RG, Detrick B, (eds) Manual of Clinical Laboratory Immunology, 6th ed. ASM Press: Washington, DC, 2002, pp 1098-1107.

6 Banks PM, Battifora H, Corson JM, et al. Incorporation of immunostaining data in anatomic pathology reports. Am J Clin Pathol 1993;99:7. 